\title{
Case Report of Coroless Tea in The Treatment of Persistent High Fever After
}

\section{Covid-19 Vaccination}

Nie Leng ${ }^{1,2 *}$, Liang Youdong ${ }^{3}$

1. Shenzhen Baiyue Gene Company, Shenzhen, China, 518110;

2. Suzhou Primreg Gene Company, Suzhou, China, 215128

3. Beijing Century Healthcare Co.,Ltd. Beijing, China, 100085

Corresponding author: nieleng@hotmail.com

Keywords: Covid-19; vaccination; fever; food; Coroless; thrombus;

\section{Abstract}

Let food be thy medicine and medicine be thy food. So is the Covid-19

medicine. Persistent high fever after the Covid-19 vaccination is one symptom.

After taking Coroless tea, the patient's body temperature returned to normal in two or three days. Timely temperature reduction is very helpful in eliminating the anxiety of the patients and their families. Coroless tea, as food, is one choice of treatment for fever after Covid-19 vaccination. 
Fever after the Covid-19 vaccination is a common symptom. So effective fever reduction method at home has reference value, especially for those with persistent high fever.

The patient was about forty years old. After the second dose of the SARS-Cov2 Vaccine in Beijing, his skin was itchy, with a high fever of $38.4^{\circ} \mathrm{C}$, and headaches for three consecutive days. The examination reported that the creatinine exceeded the upper limit, the prealbumin exceeded the upper limit, the transaminase exceeded the upper limit, the glucose exceeded the upper limit and the C-reactive protein exceeded the upper limit, indicating kidney injury, liver injury, inflammation and insulin resistance. The doctor in hospital prescribed LianHua Qingwen Keli (Chinese patent medicine) and ibuprofen. After taking it for two days, Lianhua Qingwen was ineffective in cooling down. After taking ibuprofen, the temperature dropped significantly, but the body temperature exceeded $38^{\circ} \mathrm{C}$ again after $6-8$ hours, causing headache.

On the fifth day, Coroless tea (Suzhou Primreg Gene Company) was used at home, twice a day (Primreg Product, $2.8 \mathrm{~g}$, twice the dose for severe cases of Covid-19 disease). On the afternoon of the first day after taking Coroless, the body temperature rose to $39.1^{\circ} \mathrm{C}$, and the headache was uncontrollable. After taking ibuprofen, the body temperature dropped and the headache relieved, but then he had a fever again in the middle of the night. The next morning, Coroless tea was taken at twice the dose for severely ill patients with COVID-19. In the afternoon, the body temperature rose to $39.1^{\circ} \mathrm{C}$ again. After dinner, Coroless tea 
was taken again at twice the dose for severely ill patients with COVID -19. Two days after taking Coroless tea, the body temperature was normal in the morning and Coroless reduce dose by half, and then take it for two more days. No more fever at body temperature. Hospital laboratory tests showed that creatinine returned to normal, prealbumin was normal, and glucose was normal. There is another case of patients with high fever after vaccination in Beijing. Ddimer in blood exceeded upper limit, implying a high risk of thrombosis. The body temperature returned to normal within three days after taking Coroless tea at home.

After the Covid-19 vaccination, someone may have persistent fever. Patients and their families would be very anxious if the drugs are not effective. Timely temperature reduction is very helpful in eliminating the anxiety of the patients and their families.

We believe that good medicine for Covid-19 require three points: 1 non-toxic and harmless, can be used as food; 2 effectively inhibit the corona virus infecting cells; 3 efficiently repair the damage by the virus. Coroless, effective molecule isolated from the Chinese herb Kyllinga ${ }^{[1]}$ and prepares it into tea, which meets the above three points and complies with the US FDA, EU and Chinese food standards. In Vitro experiment confirmed that it effectively prevents the Covid-19 virus from infecting cells. It has a significant effect on the repair of lung injury in severely ill Covid-19 patients in Wuhan. It is also used for COVID-19 prevention. ${ }^{[1-3]}$ Here, 
Coroless tea was used to treat adverse reactions caused by the Covid-19 vaccine to protect public health.

"Let food be thy medicine and medicine be thy food." Hippocrates once said. So is the Covid-19 medicine.

Hand in hand, human being would be free from coronavirus fear forever!

\section{Consent}

Written informed consent for publication of their clinical details and clinical data was obtained from the patient.

\section{REFERENCES}

1. Nie Leng, Liang Youdong, Yang Xinwei, Coroless, Effective Ingredient Isolated from Traditional Chinese Medicine for the Prevention and Treatment of COVID-19, J Antivir Antiretrovir, Vol.13 Iss.3 No:217;

2. Nie Leng, Liang Youdong, Yang Xinwei,,Huang Xiaohao, Wang Shuimei, Chen Jianfei, Prevention and Treatment of COVID-19 and Rehabilitation of Kidney, Liver and Cardiovascular Diseases with Kyllinga brevifolia Rottb., Medicinal Plant, 2021, 12(2):47-49;

3. Nie Leng, Liang Youdong. The application of kyllinga brevifolia rottb in the prevention and treatment of COVID-19, 2020, Patent. 